\title{
Facile Integration of Ordered Nanowires in
}

\section{Functional Devices}

Jordi Guilera ${ }^{a}$, Cristian Fàbrega ${ }^{a, b}$, Olga Casals ${ }^{b}$, Francisco Hernández-Ramírez ${ }^{a, b}$,

Shangzhou Wang ${ }^{c}$, Sanjay Mathur ${ }^{c}$, Florian Udrea ${ }^{d, e}$, Andrea De Luca ${ }^{d}$, S. Zeeshan Alie,

Albert Romano-Rodríguez ${ }^{b}, J$. Daniel Prades ${ }^{b, *}$, Joan R. Morante ${ }^{a, b, *}$

a. IREC, Catalonia Institute for Energy Research, San Adrià de Besòs, Spain.

b. Departament d'Electrònica, Universitat de Barcelona. Barcelona, Spain.

c. Institute of Inorganic Chemistry, University of Cologne. Cologne, Germany.

d. Engineering Department University of Cambridge, Cambridge, United Kingdom.

e. Cambridge CMOS Sensors Ltd, Cambridge, United Kingdom.

* Corresponding Author J. Daniel Prades, dprades@el.ub.edu

KEYWORDS: Nanowire, Nanofabrication, Devices, Electrodes, Dielectrophoresis, Gas Sensor, Photodetector, Field-Effect Device, Low Cost Integration 
ABSTRACT: The integration of one dimensional (1D) nanostructures of non-industry-standard semiconductors in functional devices following bottom-up approaches is still an open challenge that hampers the exploitation of all their potential. Here, we present a simple approach to integrate metal oxide nanowires in electronic devices based on controlled dielectrophoretic positioning together with proof of concept devices that corroborate their functionality. The method is flexible enough to manipulate nanowires of different sizes and compositions exclusively using macroscopic solution-based techniques in conventional electrode designs. Our results show that fully functional devices, which display all the advantages of single-nanowire gas sensors, photodetectors, and even field-effect transistors, are thus obtained right after a direct assembly step without subsequent metallization processing. This paves the way to low cost, high throughput manufacturing of general-purpose electronic devices based on non-conventional and high quality 1D nanostructures driving up many options for high performance and new low energy consumption devices. 


\section{INTRODUCTION}

One dimensional (1D) nanostructures offer a convenient path for the synthesis of high-quality, almost defect free, crystals of non-industry-standard semiconductor materials, such as metal oxides $(\mathrm{MOX})[1,2]$. However, the integration of these materials in functional devices, with the necessary degree of order and complexity, is an open challenge that still hampers the exploitation of their potential in full $[2,3]$. Current bottom-up approaches rely on a complex combination of positioning methods (e.g. nanomanipulation, self-assembly, di/electrophoresis, etc.) [3-5], and connecting methods (e.g. UV, Electron/Ion Beam, Soft lithographies) [6-8]. This strategy is however far from reconciling technical requisites with cost requirements of the industry.

A decade of research efforts shows that the most advantageous nanodevice configuration requires either a single (or a few) nanowires running parallel between two electrodes [9] to thus build up a well-defined conduction channel to be easily modulated by external stimuli (e.g. chemical or biological agents [10,11], light or radiation [12], external electric fields [13,14], etc.). This means explicitly avoiding random wire-to-wire conduction paths which inevitably appear in devices based on randomly oriented mats of 1D nanostructures [15].

In the particular case of metal oxide nanowire-devices, this configuration has successfully led to a high number of applications and proof-of-concept prototypes, proving their interest in high temperature and power electronics, in which highly miniaturized field-effect transistors of wideband gap semiconductor are sought after [16-18].

On the one hand, and regarding gas detection applications, nanowire-based metal oxide sensors $[19,20]$ display important advantages only accessible in ordered configurations [15]. Firstly, the large surface-to-volume ratio of nano-sized materials provides a systematic way to increase the influence of surface phenomena, such as the response to gases [21]. Secondly, nanowires are 
typically enclosed by well-terminated surfaces in well-defined crystal orientations, offering a reduced set of interaction sites with gases that render better reaction control, and in general good signal stability [15]. Thirdly, the response dynamics of one or a few nanowires is theoretically faster, if compared to porous layers made of nanoparticles, since all the sensing material is simultaneously exposed to the changes in the gaseous environment [15]. Last but not least, the self-heating effect [22] of nanowire devices can be used to dramatically reduce the power consumption typical of standard MOX sensors (from $\mathrm{mW}$ in microdevices to $\mu \mathrm{W}$ in self-heated nanowires[23]) with faster heating/cooling times (in the ranges of ms [24]), in temperature modulated applications.

On the other hand and as far as photodetection applications go, metal oxide nanowires show high intrinsic photoconductive gain values [12,25] mainly linked to the high crystallinity and ultimately caused by the electron-hole separation processes at the surface [26]). Additionally, in contrast with structures based on randomly oriented materials, parallel nanowire arrangements enable uniform light absorption through all the photoconductor material. All in all, MOX nanodevices offer a big potential to build up highly-sensitive photodetectors [27].

Anyhow, all these applications are mostly limited by the lack of simple, cost effective and scalable methods to transfer, precisely position and interconnect nanowires. Among the most promising approaches, macroscopically driven dielectrophoresis [5] seems to address the manipulation concerns in a cost effective manner; but so far, only for selected materials $[5,28]$, and in highly specialized electrode configurations $[4,18]$ and usually followed by a contact fabrication step.

Here, we present a facile and high throughput methodology to build up ordered arrangements of metal oxide nanowires, between a pair of conventional electrodes without the need of any 
subsequent micromanipulation, lithography or post processing technique. Our results prove that the method, based on dielectrophoresis, is flexible enough to integrate different types of metal oxide nanowires (such as $\mathrm{SnO}_{2}, \mathrm{ZnO}, \mathrm{TiO}_{2}$, etc.) and attain the functionalities and the advantages expected from individual nanowires.

\section{METHODS}

\subsection{Materials}

To demonstrate the flexibility of our method, three different metal oxide nanowires were used as a proof of concept.

First, crystalline $\mathrm{SnO}_{2}$ nanowires were produced by chemical vapor deposition (CVD) of a molecular precursor $\left[\mathrm{Sn}\left(\mathrm{O}^{t} \mathrm{Bu}\right)_{4}\right]$, catalyzed with $\mathrm{Au}$ on top of a $\mathrm{Si}$ wafer and following a procedure reported elsewhere [29-31]. The products were longer than $15 \mu \mathrm{m}$ with typical diameters of $30 \pm 10 \mathrm{~nm}$.

Second, $\mathrm{ZnO}$ nanowires were obtained by a hydrothermal process reported earlier [32]. In brief, an $80 \mathrm{~nm}$ thick seed layer of $\mathrm{ZnO}$ was sputtered on indium-doped tin oxide (ITO) glass. The ITO glass was then placed in the growth solution consisting of $0.02 \mathrm{M}$ zincnitrate, $0.015 \mathrm{M}$ hexamethylenetetramine (HMTA), $0.004 \mathrm{M}$ polyethyleneimine (end-capped, molecular weight $800 \mathrm{~g} / \mathrm{mol}$ ) and $0.024 \mathrm{M}$ ammonium hydroxide. The solution was heated to $88^{\circ} \mathrm{C}$, and after 3 hours, $\mathrm{ZnO}$ nanowires of about $6 \mu \mathrm{m}$ in length with diameters from 120 to $180 \mathrm{~nm}$ were obtained. The resulting $\mathrm{ZnO}$ arrays were finally rinsed with Mili-Q water and dried in nitrogen flow.

Third, $\mathrm{TiO}_{2}$ nanowires were obtained by ALD conformal coating of silicon nanowires growth (typical diameter of $100 \mathrm{~nm}$ and 5-7 $\mu \mathrm{m}$ in length) by CVD with a conventional method [33]. 
Tetrakis(dimethylamino)titanium(IV) (TDMAT) was used as a titanium source during the ALD process and water as an oxidizing agent. Growth temperature was fixed at $250^{\circ} \mathrm{C}$ with a sequence of 5 seconds of TDMAT followed by 5 seconds of water. A total of 2500 cycles were applied to obtain $100 \mathrm{~nm}$ of $\mathrm{TiO}_{2}$, corresponding to a growth rate of $0.4 \AA /$ cycle.

As for the substrates, commercial microhotplates $(\mu \mathrm{HP})$ with Au Inter Digital Electrodes (IDE) from Cambridge CMOS Sensors (www.ccmoss.com) were used. These devices were originally designed to accommodate a gas sensitive layer across two IDE electrodes. No modification of the general-purpose IDE design was made to facilitate the here-proposed integration method. Gold is inert to oxidation and the rough $\mathrm{Au}$ surface provides better attachment to the later deposited nanowire, according to preliminary tests. The IDEs spanned a circular area of $250 \mu \mathrm{m}$ in diameter and the gap between the IDE fingers was $10 \mu \mathrm{m}$. In order to reach the optimum operating temperatures, the devices also integrate a microheating element made of tungsten, and buried under the IDEs within $\mathrm{SiO}_{2}$ insulation, following an architecture reported elsewhere [34]. Unlike polysilicon heaters, the tungsten heaters offer a much higher stability at high temperatures. The circular $\mathrm{SiO}_{2}$ insulating membrane obtained by Deep Reactive Ion Etching (DRIE) embedding the IDEs/heater had a diameter of about $640 \mu \mathrm{m}$. IDEs, and associated bond pads, were manufactured post CMOS sequence. The maximum temperature the $\mu \mathrm{HP}$ can reach is about $700^{\circ} \mathrm{C}$, while the power consumption is only about $55 \mathrm{~mW}$ at $450^{\circ} \mathrm{C}$. In this work, the heating element was solely used for test and calibration purposes.

\subsection{Integration methodology}

The as-grown nanowires were first removed from their original substrates by means of sonication. To that end, the substrates were fully immersed in isopropanol and then submitted to 
an ultrasonic bath for a few minutes. Power and duration of the bath were adjusted to obtain dispersions with suitable nanowire concentrations in all cases.

For the nanowire integration in the $\mu \mathrm{HPs}$ and subsequent alignment between the IDEs, dielectrophoretic forces were used [5] applying an $\mathrm{AC}$ square signal (15 V peak-to-peak) to the electrodes. One or more drops of the nanowire's dispersion were deposited on top of the IDE using a microsyringe. The frequency of the signal was then adjusted for each material to achieve an optimum coverage and alignment of nanowires.

After the deposition and induced alignment of the nanowires across the IDE, the resulting devices were kept at $100^{\circ} \mathrm{C}$ for 2 hours to facilitate the full evaporation of the solvent residues.

\subsection{Device test and characterization methods}

The resulting devices were first electrically tested; Current-Voltage (I-V) curves were acquired with a dual-channel Keithley SourceMeter Unit (SMU) model 2600 and custom-made LabView software. Shielded coaxial wires, fixings and test chambers were used to minimize noise in the electrical measurements due to the high resistance of some devices. In temperature controlled experiments, the microheater of the $\mu$ HPs was also powered by the second SMU channel of the same instrument. The calibration curves provided by the manufacturer and in-house validated were used to estimate the temperature reached by the $\mu \mathrm{HP}$. The direct comparison of the device resistance values at different microheater operating conditions and those obtained as a function of increasing current level without the heater was used to estimate the equivalent temperature reached due to the self-heating effect $[23,35]$.

In gas sensing experiments, accurate gaseous environments were maintained inside the test chamber with mass flow controllers mixing dry synthetic air (SA, 21\% $\mathrm{O}_{2}$ and $79 \% \mathrm{~N}_{2}$ ), and $\mathrm{CO}$ (100 ppm $\pm 1 \%$ in SA). These lab-class gases contained less than $5 \mathrm{ppm} / \mathrm{vol}$ of $\mathrm{C}_{\mathrm{n}} \mathrm{H}_{\mathrm{m}}$ and $\mathrm{H}_{2} \mathrm{O}$. 
For experiments under illumination, a Hammamatsu LC8 light source with a type [-01] Hg-Xe lamp enhanced for the line at $\lambda=365 \mathrm{~nm}$ was used. UV light intensity impinging on the devices was determined with the help of a thermopile detector (FieldMax-TOP).

Ten devices of each type were fabricated and tested showing the typical responses presented in the following sections, in a reproducible manner for more than 12 weeks.

\section{RESULTS AND DISCUSSION}

\subsection{Device fabrication}

The fabrication process consisted of three steps (Figure 1.a): (1) transfer of nanowires from the growth substrate to a liquid suspension, (2) drop casting on top of the Inter Digital Electrodes (IDE) and, simultaneously, (3) nanowire positioning and alignment using dielectrophoretic forces. All these three steps can be controlled macroscopically and are scalable to the necessary production level in industry in a cost effective manner.

Going into details, nanowires (NW) were first removed from the substrate by direct sonication in isopropanol. The materials properties (length, stiffness, density, etc.) determined the optimum sonication time and necessary power to achieve a suitable concentration of dispersed nanowires. As a rule of thumb, concentrations in the order of $100 \mathrm{NW} / \mu 1$ were appropriate in our experiments. Care was taken to prevent excessive nanowire fracture during the sonication step, as the final nanowires length had to be comparable to the IDE spacing.

Figures 1.b, 1.c and 1.d show the devices after the alignment step of $\mathrm{SnO}_{2}, \mathrm{ZnO}$ and $\mathrm{TiO}_{2}$ nanowires respectively. Frequencies of $10 \mathrm{kHz}, 4 \mathrm{MHz}$ and $5 \mathrm{MHz}$ were applied in these three cases. Remarkably, the process was successful, independently on the materials composition, dimensions and polar nature. It is noteworthy that the reported conditions depend on the materials, solvent and electrode design used. Therefore, the optimization of the process in a 
different set up will certainly require different parameters that could be optimized according to the extensive literature [3-5].

In all cases, nanowires concentrated in the inter-electrode region aligned parallel to each other, and perpendicular to the electrode edges. Finite elements simulations with Accelrys COMSOL Multiphysics 4.3 of the electric field distribution across the two electrodes (Figure 1.e) showed that the nanowires mainly aligned parallel to the direction of the local electric field and concentrated in the regions with highest field strengths, which was fully determined by the layout of the commercial electrodes used.

This validates (1) that dielectrophoresis is a suitable macroscopic technique to manipulate and align nanowires, and (2) that the fabrication methodology is flexible enough to be applied to different kinds of metal oxides (3) without the need for a special electrode design.

Further experiments showed that fine tuning of the number of nanowires positioned across the IDEs could be achieved by adjusting the concentration of nanowires in the dispersion, the number of drops deposited and the applied $\mathrm{AC}$ frequencies used, as function of the material and geometry.

\subsection{Electrical properties}

According to SEM micrographs (Figure 1.b to 1.e), the nanowires bridged the insulating gap across the IDE after the positioning step. To assess the electrical quality of the contacts, currentvoltage (I-V) curves were acquired. Figures 2.a, 2.b and 2.c show that current flowed through the nanowires in all cases. The average $\mathrm{DC}$ resistance was $\mathrm{R}_{\mathrm{SnO} 2}=10.5 \pm 0.1 \mathrm{M} \Omega$, $\mathrm{R}_{\mathrm{ZnO}}=42 \pm 1 \mathrm{M} \Omega$ and $\mathrm{R}_{\mathrm{TiO} 2}=0.72 \pm 0.05 \mathrm{M} \Omega$, at room temperature $\left(25^{\circ} \mathrm{C}\right)$. Resistance values up to $10 \mathrm{~T} \Omega$ were measured between electrodes after depositing and drying several drops of pure isopropanol, confirming that the observed conductance can only be assigned to the nanowires. 
For $\mathrm{SnO}_{2}$, an entirely Ohmic behavior was observed, whilst in the case of $\mathrm{ZnO}$ and $\mathrm{TiO}_{2}$ the $\mathrm{I}-\mathrm{V}$ curves were nonlinear in the range of tested temperatures. This effect is explained by the nature of the metal/semiconductor contacts that develops between the $\mathrm{Au}$, and the metal oxides, which is reported to be nearly Ohmic for as-grown $\mathrm{SnO}_{2}$ [36], and rectifying for $\mathrm{ZnO}$ [37] and $\mathrm{TiO}_{2}[38]$ due to the formation of Schottky barriers. In fact, similarly high resistance contact values are found in single wire devices, fabricated with certain nanolithography methods [39].

To further verify the characteristics of the conduction path built by the nanowires, the dependence of the device resistance as function of the temperature was measured, observing a clear drop in all cases as temperature increased (Figure 2.a to 2.c). Interestingly, after this heating process, no change in the device resistance at room temperature was observed, indicating that the electrical contact was already stable after solvent evaporation.

It is clear, however, that these resistance values were high, considering the typical resistance of a single nanowire $[25,39,40]$ and taking into account the high number of nanowires connected in parallel. This fact may indicate (1) that not all the nanowires were effectively contacted and/or (2) the nanowire/metal contacts quality was very poor (which is plausible in case of different materials in soft contact [41]).

Concerning contact stability, specific experiments showed that the resistance baseline of the devices displayed less than $2 \%$ drift after 2 weeks of continuous operation at $200^{\circ} \mathrm{C}$. Additionally, current-voltage curves acquired repeatedly on the same device for 15 days, showed differences of less than $0.1 \%$ in average. Both facts are evidences that the contacts are stable and solid. In fact, similar contacts are present in commercial metal oxide sensors based fabricated by direct screen printing of nanoparticles slurries. In such commercial devices no further metallization is used. 
Nevertheless, our results show that this integration methodology is suitable to effectively connect nanowires with metal electrodes, without the need of any subsequent metallization steps. As shown in the following sections, the electrical continuity achieved, despite clearly improvable, is sufficient to take advantage of the favorable properties of single-wire devices.

\subsection{Application to gas sensing}

In self-heating operation mode, the current applied to probe the resistance of a nanowire dissipates enough power (in the $\mu \mathrm{W}$ range) to heat up the nanostructure to temperatures sufficiently high (hundreds of ${ }^{\circ} \mathrm{C}$ ) to thus activate the thermal response to gases [23].

Figure 3.a shows that this phenomenon is clearly noticeable in $\mathrm{SnO}_{2}$-based devices, which is the most attractive material for gas sensing applications. At very low probing currents $(0.1 \mathrm{nA})$, no gas response was observed to different concentrations of CO diluted in dry SA. As the probing current increased, the modulation of the resistance appeared in presence of the target gas (CO); e.g. a factor 6 at $[\mathrm{CO}]=100 \mathrm{ppm}$. Using the resistance dependence of the nanowire material with temperature, equivalent operating temperatures up to $200^{\circ} \mathrm{C}$ could be estimated. At this temperature, the devices detected $\mathrm{CO}$ concentrations much lower than the ones shown here (down to $5 \mathrm{ppm}$ ). In all cases (Figure 3.b), the resistance modulation followed the expected power law $\mathrm{R}_{\mathrm{SA}} / \mathrm{R}_{\mathrm{CO}} \propto[\mathrm{CO}]^{\mathrm{n}}$ typical for MOX sensors [41]. At the optimum temperatures for sensing, the exponent was estimated to be $\mathrm{n}=+0.5 \pm 0.1$ in agreement with the values predicted by theoretical models for the interaction between $\mathrm{SnO}_{2}$ and $\mathrm{CO}$ predict $\left(\mathrm{n}_{\text {theory }}=+1 / 2 \quad[41]\right)$, suggesting that the here reported devices operate following the conventional sensing mechanisms in MOX sensors.

The responses obtained at equivalent temperatures were compared; fixed either with selfheating or using the calibrated microheater (Figure 3.c). In both experiments, equivalent 
responses were obtained but their dynamics were clearly different. This fact may originate from differences in the local temperature achieved by each nanowire in self-heating mode, which leads to an overall response signal that averages the dynamics of nanowires operating at different temperatures [42].

Concerning power consumption, our devices exhibited figures in the order of $200 \mu \mathrm{W}$ to reach temperatures of $200^{\circ} \mathrm{C}$, which is remarkably low and comparable to those obtained with complex devices based on single nanowires [23]. It goes without saying that this feature is an additional step forward towards heater-free solid-state sensor technology such as the one proposed by Monereo et al. [42].

Besides, reproducing pulsed operation mode was also feasible as shown in Figure 3.d. The response time ${ }^{1}$ of the resistance modulation to probing current changes (i.e. temperature) was in the order of tens of ms, both in the heating and cooling transients. These values are again comparable to the figures obtained with individual nanowires [43], sufficiently fast to study sudden surface-gas interactions [24], and clearly much faster than values reported for gas sensor based on thin films [44].

All these results demonstrate that our devices possess all the advantages commonly attributed to gas sensors based on individual nanowires; namely: (1) large responses, (2) good signal stability, (3) fast response dynamics, and (4) ultra-low power consumption in fast self-heating operation mode.

\subsection{Application to photodetectors}

${ }^{1}$ Resistance stabilization to thermal changes was fitted to an exponential law for the heating and cooling semi periods. Response times were defined as the exponential time constant of the transient. 
$\mathrm{ZnO}$ is one of the most promising materials for optoelectronic applications in the ultraviolet range [12], with remarkably high sensitivity when shaped in the form of nanowires [25-27]. According to the literature, an ordered arrangement of a few nanowires is necessary to reach such high values [45].

Figure 4.a shows the typical photocurrent generated in one of our $\mathrm{ZnO}$-based devices upon exposure to UV light pulses, at two different bias conditions. Clearly (Figure 4.b), the photocurrent $\left(\mathrm{I}_{\mathrm{ph}}\right)$ scales proportionally to the flux of photons $\left(\Phi_{\mathrm{ph}}\right)$ and as function of the bias voltage $\left(\mathrm{V}_{\text {bias }}\right)$, as expected for a photoconductor device [45]. This behavior leads to normalized photoconductive gains ${ }^{2}\left(\mathrm{~g}_{\mathrm{ph}}\right)[45]$ in the order of $\mathrm{g}=10^{-7} \mathrm{~m}^{2} / \mathrm{V}$, to be compared with the best values in the literature $\mathrm{g}_{\mathrm{Lit}}{ }^{\mathrm{NW}}=10^{-5}$ to $10^{-6} \mathrm{~m}^{2} / \mathrm{V}$ reported for single-wire devices [27,45], and $\mathrm{g}_{\mathrm{Lit}}^{\mathrm{TF}}=10^{-8} \mathrm{~m}^{2} / \mathrm{V}$ reported for thin film devices [46].

From a dynamic perspective, our devices $(\tau \sim 10 \mathrm{~s})$ were slightly faster than conventional single-wire devices $\left(\tau_{\mathrm{Lit}}{ }^{\mathrm{NW}} \sim 30 \mathrm{~s}\right.$ [45]), but still much slower than thin film ones $\left(\tau_{\mathrm{Lit}}{ }^{\mathrm{TF}} \sim 1.5 \mu \mathrm{s}\right.$ [46]). These differences can be fully explained by taking into account the strong influence of surface-mediated electron-hole separation effects in nano-size materials [26]: charge separation slows down the electron-hole recombination times (and thus the response times), leading to larger photocurrent values.

Again, the optoelectronic performance of the devices herein implemented is (1) sufficient for UV photodetection applications, (2) fully comparable to that of devices made with much more

${ }^{2}$ For the calculations of $g_{p h}$, according to the definitions in Ref. [45], an average density of 0.4 nanowire $/ \mu \mathrm{m}$ along the electrode edges and nanowire diameters of $150 \mathrm{~nm}$ were assumed to estimate the width of the light collection area (W). As for the inter electrode distance (L) manufacturer specifications $(10 \mu \mathrm{m})$ were used. This way, after geometry (W,L) and bias voltage $\left(\mathrm{V}_{\text {bias }}\right)$ correction, $\mathrm{g}_{\mathrm{ph}}$ estimations at different $\mathrm{V}_{\text {bias }}$ were fully consistent. 
complex fabrication methods (e.g. single-wire devices), and (3) unexpectedly good, taking into account the moderate quality of the wire-to-metal and wire-to-wire contacts.

\subsection{Application to Field Effect Transistors}

Our results indicate that the fabrication method is even suitable to build up field-effect devices as well. To that end, the pair of IDE electrodes were used as source (label S) and drain (label D), to define a conduction channel along all the parallel aligned nanowires. The tungsten microheater, which was embedded in the substrate under the nanowires in a dielectric $\mathrm{SiO}_{2}$ layer, was used as gate (label G) terminal. The current-voltage characteristics of these FET devices made of $\mathrm{ZnO}$ nanowires show (Figure 5.a) that the conduction along nanowires can be effectively modulated by a gate voltage $\mathrm{V}_{\mathrm{G}}$. To determine the efficiency of the gating behavior, transconductance measurements $\left(\mathrm{I}_{\mathrm{SD}}\right.$ vs $\left.\mathrm{V}_{\mathrm{G}}\right)$ were obtained at a variety of $\mathrm{V}_{\mathrm{SD}}$ values (Figure 5.b), observing a typical n-type behaviour. The threshold voltages were around $-4 \mathrm{~V}$ for all $\mathrm{V}_{\mathrm{SD}}$ values. The on-off current ratio $\left(\mathrm{I}_{\mathrm{ON}} / \mathrm{I}_{\mathrm{OFF}}\right)$ was $\sim 30$ at $\mathrm{V}_{\mathrm{SD}}=5 \mathrm{~V}$, which is comparable to other results reported in the literature with more complex fabrication approaches $[18,47]$.

From the transconductance plots, the mobility $(\mu)$ and carrier concentration $\left(\mathrm{n}_{\mathrm{e}}\right)$ can be estimated by using the expressions for a conduction channel built in one single nanowire [14],

$$
\begin{aligned}
& \frac{\mathrm{dI}_{\mathrm{Sd}}}{\mathrm{dV}_{\mathrm{G}}}=\frac{\mu \mathrm{C}}{\mathrm{L}^{2}} \\
& \mathrm{C}=\frac{2 \pi \varepsilon_{0} \varepsilon_{\mathrm{r}} \mathrm{L}}{\ln \left(\frac{4 \mathrm{~h}}{\mathrm{~d}}\right)} \\
& \mathrm{n}_{\mathrm{e}}=\frac{\mathrm{V}_{\mathrm{th}} \mathrm{C}}{\mathrm{q} \pi\left(\frac{\mathrm{d}}{2}\right)^{2} \mathrm{~L}}
\end{aligned}
$$

where $\mathrm{C}$ is the surface capacitance of a nanowire, $\varepsilon_{\mathrm{r}}$ is the dielectric constant $\left(\varepsilon_{\mathrm{r}}=4\right)$ and $\mathrm{h}$ the thickness $(3 \mu \mathrm{m})$ of the $\mathrm{SiO}_{2}$ gate dielectric, $\mathrm{V}_{\text {th }}$ is the threshold voltage, $\mathrm{d}$ is the nanowire diameter $(150 \mathrm{~nm}), \mathrm{q}$ is the electron charge, and $\mathrm{L}$ is the length of the nanowire channel. The 
IDE spacing $(10 \mu \mathrm{m})$ has been taken as L, since several nanowires (two or three, typically) are needed to bridge across both IDEs. Our system is composed of several (N) nanowires connected in parallel. Thus, assuming that the total current ( $\left.\mathrm{I}_{\mathrm{SD} \text { Total }}\right)$ flows evenly through them, one can rewrite eq.(1), (2), and (3) as,

$$
\begin{aligned}
& \mathrm{I}_{\text {SD Total }}=\sum_{\mathrm{i}=0}^{\mathrm{N}} \mathrm{I}_{\mathrm{i}}=\mathrm{N} \cdot \mathrm{I}_{\mathrm{i}} \stackrel{\text { eq.(1) }}{\longrightarrow} \frac{1}{\mathrm{~N}} \frac{\mathrm{dI}_{\mathrm{i}}}{\mathrm{dV}_{\mathrm{G}}}=\frac{\mu \mathrm{C}_{\text {Total }}}{\mathrm{L}^{2}} \\
& \mathrm{C}_{\text {Total }}=\sum_{\mathrm{i}=0}^{\mathrm{N}} \mathrm{C}_{\mathrm{i}}=\mathrm{N} \cdot \mathrm{C}_{\mathrm{i}} \\
& \mathrm{n}_{\mathrm{e}}=\frac{\mathrm{V}_{\mathrm{th}} \mathrm{C}_{\mathrm{i}}}{\mathrm{q} \pi\left(\frac{\mathrm{d}}{2}\right)^{2} \mathrm{~L}}
\end{aligned}
$$

where $I_{i}$ and $C_{i}$ are the current and the surface capacitance of each single nanowire, which are assumed to be identical.

Figure 5.c shows the carrier mobility $(\mu)$ as a function of the number of nanowires $(N)$ effectively connected between the IDE, calculated with eq.(4), (5) and (6) and the slope of the $\mathrm{I}_{\mathrm{SD}} \mathrm{Vs} \mathrm{V}_{\mathrm{G}}$ plot at $\mathrm{V}_{\mathrm{SD}}=4 \mathrm{~V}$ (Figure 5.b). Carrier mobilities ranging from 3000 to $0.3 \mathrm{~cm}^{2} \mathrm{~V}^{-1} \mathrm{~s}^{-1}$ have been estimated, assuming that 1 to 100 nanowires were connected. Considering the typical mobility values for $\mathrm{ZnO}$ nanowires reported in the literature (from 2 to $30 \mathrm{~cm}^{2} \mathrm{~V}^{-1} \mathrm{~s}^{-1}$ [48]), one can estimate that 10 to 40 nanowires were effectively connected. This is plausible taking into account SEM images that show that not all the nanowires paths completely connect both sides of the IDE. Moreover, the previous calculations led to carrier concentration values of $n_{e}=(1.3 \pm$ $0.7) \cdot 10^{17} \mathrm{~cm}^{-3}$, in agreement with previously reported values [14].

These final results open the door to develop in future works field-effect devices based on this simple assembly technique.

\section{CONCLUSIONS}


In summary, a simple approach to integrate metal oxide nanowires in electronic devices based on dielectrophoretic alignment has been presented. The method is flexible enough to manipulate different materials and nanowire dimensions with conventional electrode designs using exclusively macroscopic solution-based techniques.

In fact, our results demonstrate that fully functional devices, which display all the advantages of single-nanowire-based gas sensors, photodetectors, and field-effect transistors, are obtained right after the assembly step, and no additional metallization is required. This means that most of the design constrains typically imposed and attributed to dielectrophoretic methods (e.g. special needle-like electrode designs, post-metallization steps, etc.) [3-5] can be relaxed for many applications. Additionally, the method displays potential to address other applications in which multi-nanowire configurations are much sought after, such as electrocatalysis or photoelectrocatalysis.

This represents an important improvement in the race for solving the challenge of integrating nanomaterials into microdevices, and opens up new possibilities for low cost manufacturing of general-purpose electronic and transducer devices based on high quality 1D nanostructures, ranging from simple metal oxide nanowires to more complex (core shell, decorated, etc.) structures. 


\section{ACKNOWLEDGEMENTS}

The research leading to these results has received funding from the European Research Council under the European Union's Seventh Framework Programme (FP/2007-2013) / ERC

Grant Agreement n. 336917, the EU SOI HIT project $n^{\circ} 288481$, the EU WIROX project $n^{\circ}$ PIRSES-GA-2011-295216, the EU COST Action EuNetAir and the Spanish National Projects NAMIRIS (TEC2010-21357-C05-05), TEMIN-AIR (TEC2013-48147-C6-1-R) and MULTICAT (CSD2009-00050). J.D. Prades acknowledges the support from the Serra Húnter Programme. 


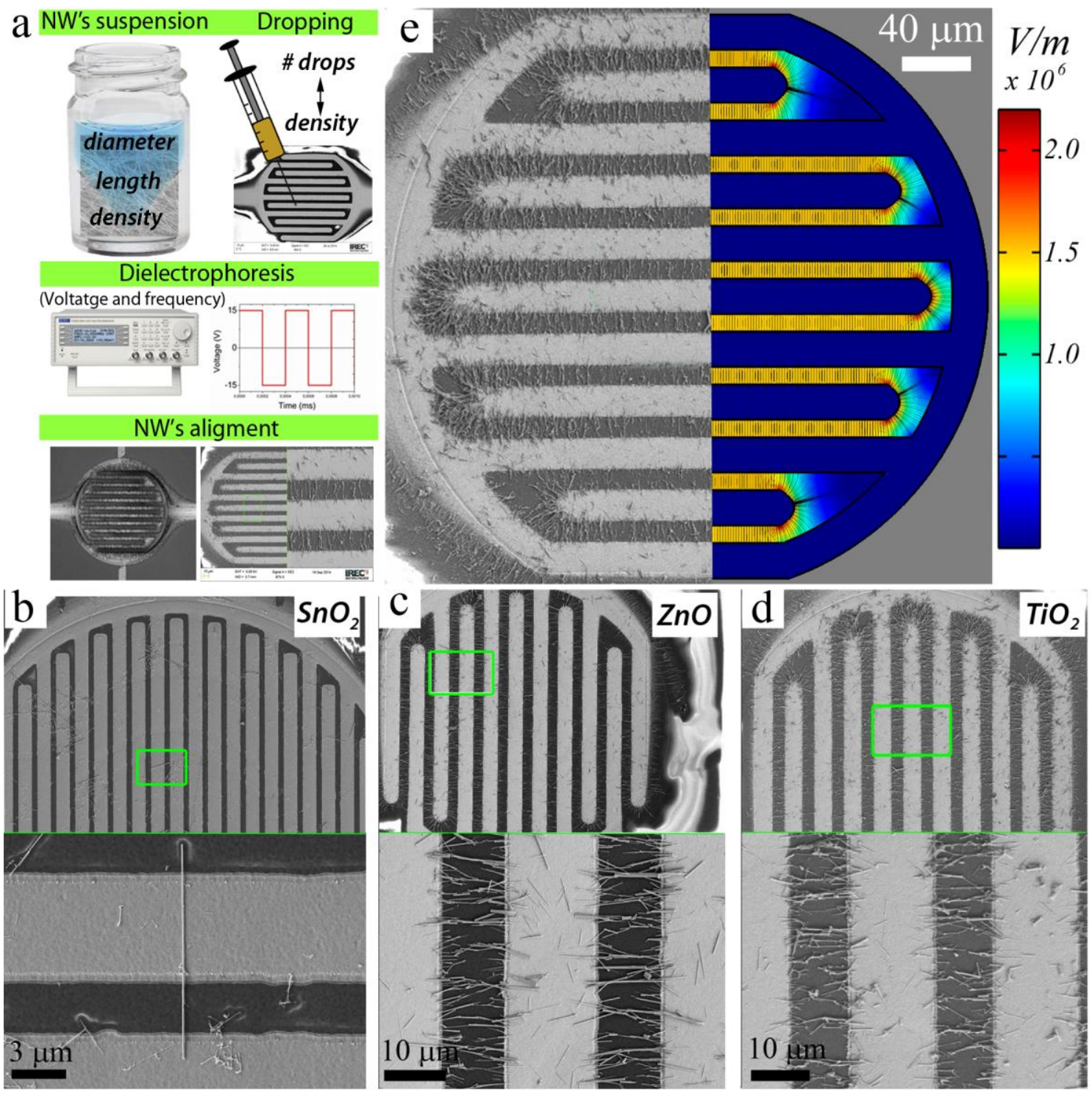

Figure 1. (a) Fabrication process steps, including: nanowire suspension preparation, drop transfer to the final substrate, and nanowire alignment with dielectrophoretic forces. This facile methodology was be successfully applied to multiple metal oxide nanowires, such as (b) $\mathrm{SnO}_{2}$, (c) $\mathrm{ZnO}$, (d) $\mathrm{TiO}_{2}$, etc. (e) According to Finite Elements simulations, the nanowire arrangement (in concentration and direction) follows the electric field distribution imposed by the electrode (IDE) geometry. 

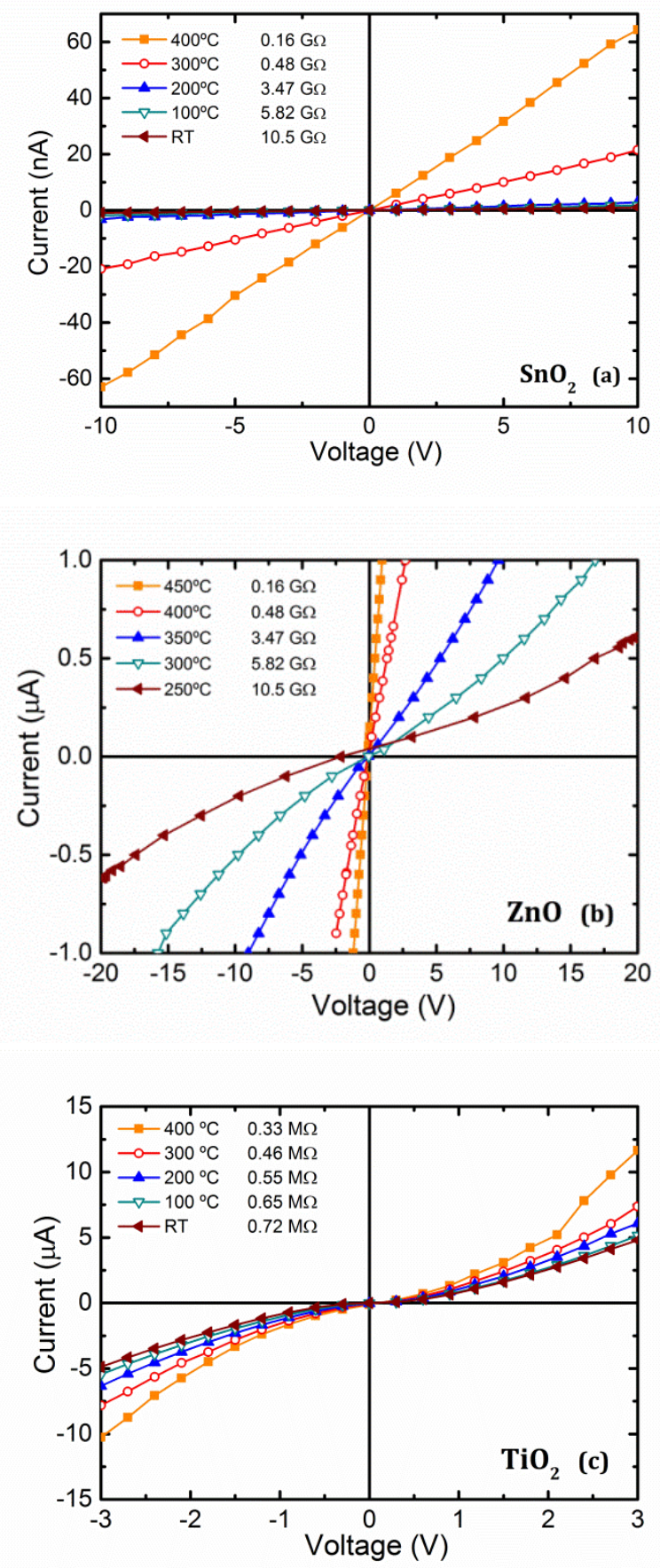

Figure 2. Typical Current-Voltage characteristics of the three kinds of devices produced: (a) $\mathrm{SnO}_{2}$, (b) $\mathrm{ZnO}$, and (c) $\mathrm{TiO}_{2}$. Resistance dependence with temperatures is consistent with the semiconductor nature of the nanowires. 

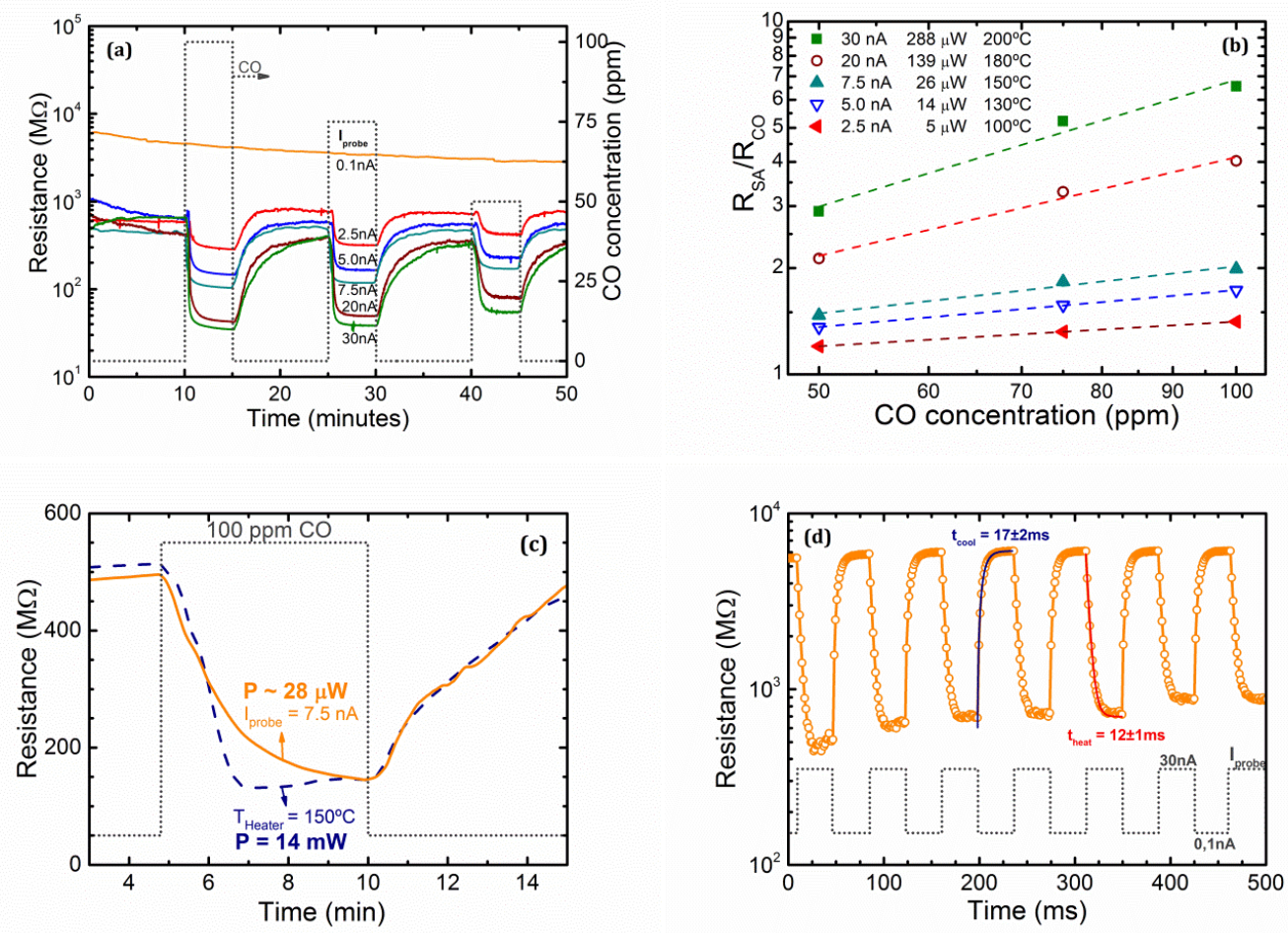

Figure 3. (a) Resistance evolution in presence of several $\mathrm{CO}$ concentrations, recorded at different probing current levels. (b) Summary of the device response (defined as $R_{\mathrm{SA}} / \mathrm{R}_{\mathrm{CO}}$ ) as a function of the carbon monoxide concentration [CO] at different probing currents in self-heating operation. The corresponding power dissipation and equivalent temperature are indicated. (c) Comparison of the responses to $100 \mathrm{ppm}$ of $\mathrm{CO}$ diluted in dry SA obtained in equivalent conditions with self-heating and with an external heater. (d) Resistance changes in response to variations of the probing current (pulsed self-heating). Exponential fittings and time constants are shown. 

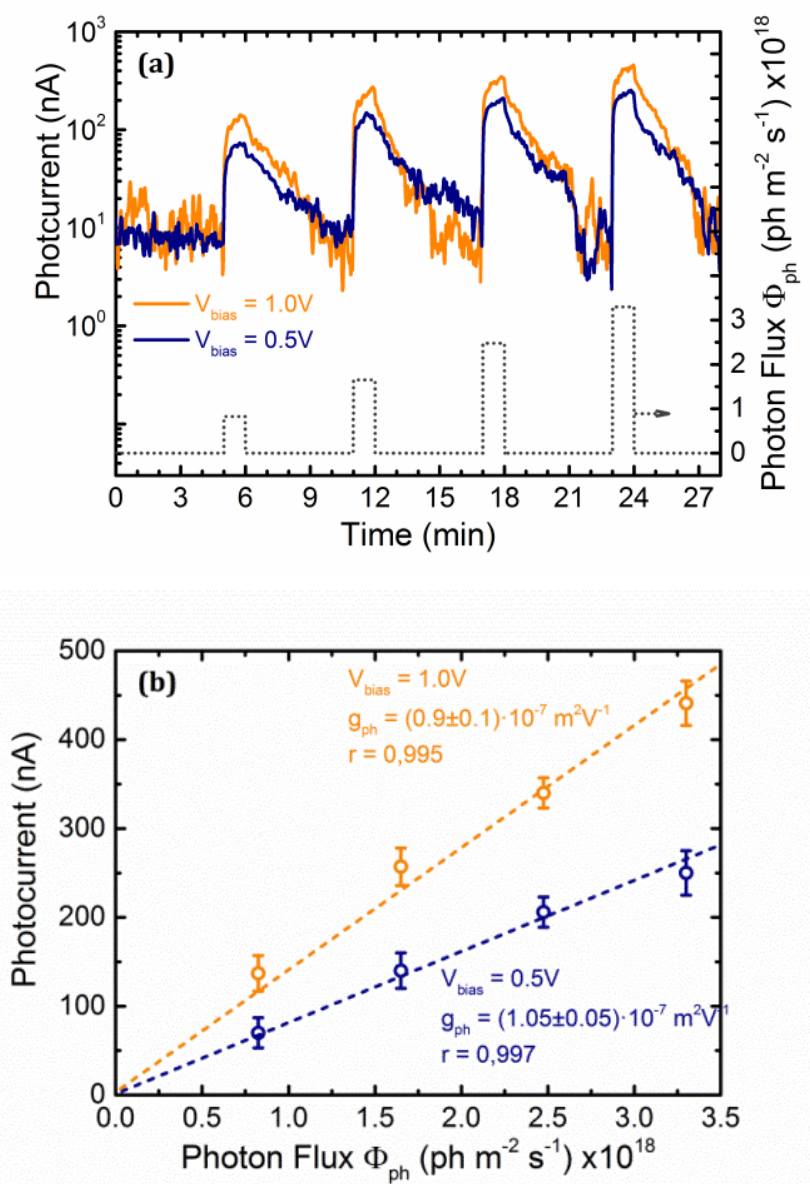

Figure 4. (a) Photocurrent generated at different light intensities $\left(\Phi_{\mathrm{ph}}\right)$ and bias voltages $\left(\mathrm{V}_{\text {bias }}\right)$. (b) Summary of the photocurrent response as a function of photon flux $\left(\Phi_{\mathrm{ph}}\right)$, where the slope is a direct measurement of $\mathrm{g}_{\mathrm{ph}}$, after geometry $(\mathrm{W}, \mathrm{L})$ and bias voltage $\left(\mathrm{V}_{\text {bias }}\right)$ correction [45]. 

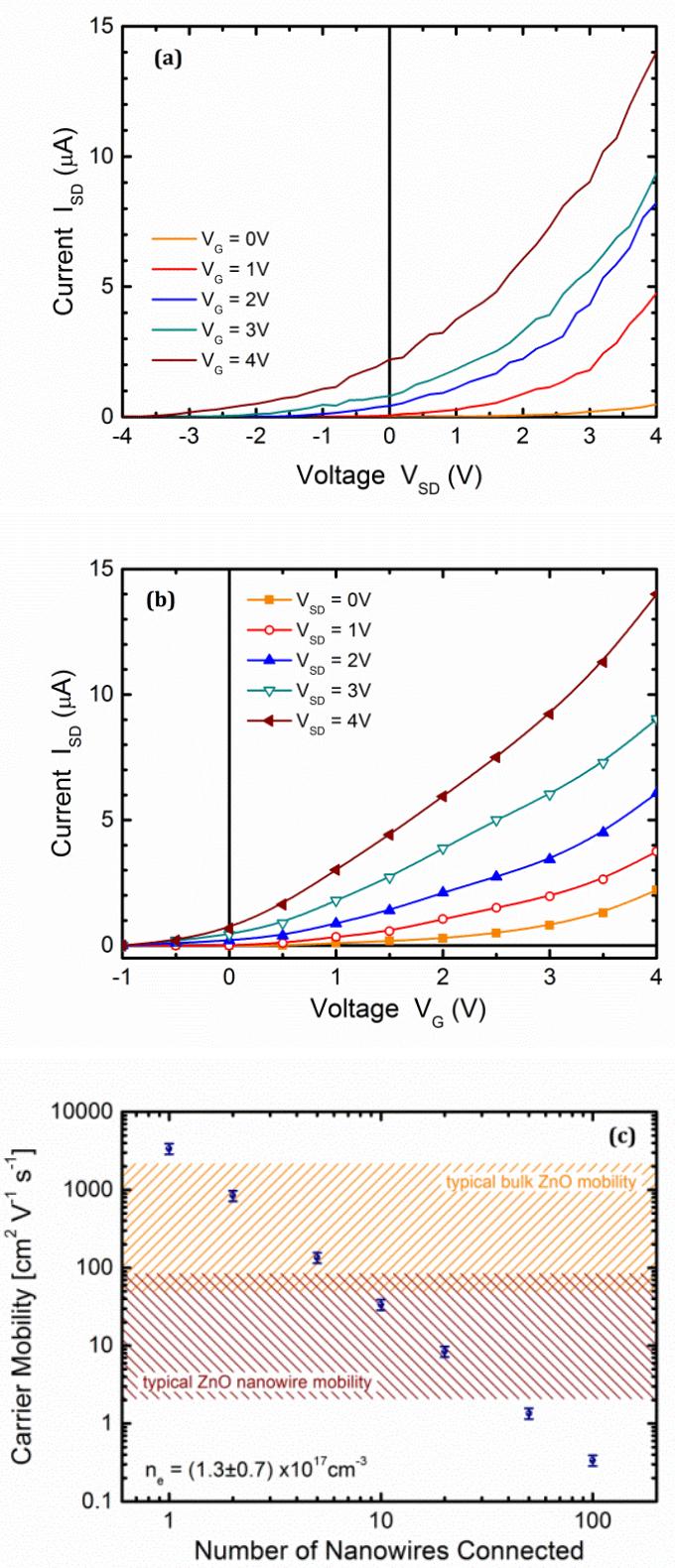

Figure 5. (a) Current $\mathrm{I}_{\mathrm{SD}}$ vs voltage $\mathrm{V}_{\mathrm{SD}}$ curves recorded at different gate voltages from 0 to $4 \mathrm{~V}$. (b) Current $I_{S D}$ vs gate voltage $V_{G}$ of the same device measured at bias voltage from 0 to $4 \mathrm{~V}$. (c) Estimated carrier mobility $\mu$ as a function of number $\mathrm{N}$ of nanowires effectively connected across the IDE. Typical $\mathrm{ZnO}$ mobility values for bulk and nanowires are marked as hashed regions [48]. 


\section{REFERENCES}

[1] J. Hu, T.W. Odom, C.M. Lieber, Chemistry and Physics in One Dimension: Synthesis and Properties of Nanowires and Nanotubes, Acc. Chem. Res. 32 (1999) 435-445. doi:10.1021/ar9700365.

[2] S. Barth, F. Hernandez-Ramirez, J.D. Holmes, A. Romano-Rodriguez, Synthesis and applications of one-dimensional semiconductors, Prog. Mater. Sci. 55 (2010) 563-627. doi:10.1016/j.pmatsci.2010.02.001.

[3] M.C.P. Wang, B.D. Gates, Directed assembly of nanowires, Mater. Today. 12 (2009) 3443. doi:10.1016/S1369-7021(09)70158-0.

[4] S. Kumar, S. Rajaraman, R.A. Gerhardt, Z.L. Wang, P.J. Hesketh, Tin oxide nanosensor fabrication using AC dielectrophoretic manipulation of nanobelts, Electrochim. Acta. 51 (2005) 943-951.

[5] E.M. Freer, O. Grachev, X. Duan, S. Martin, D.P. Stumbo, High-yield self-limiting singlenanowire assembly with dielectrophoresis., Nat. Nanotechnol. 5 (2010) 525-530. doi:10.1038/nnano.2010.157.

[6] S.B. Cronin, Y. Lin, O. Rabin, M. Black, J. Ying, M.S. Dresselhaus, et al., Making electrical contacts to nanowires with a thick oxide coating, 653 (2002). doi:10.1088/0957$4484 / 13 / 5 / 322$.

[7] G. De Marzi, D. Lacopino, a. J. Quinn, G. Redmond, Probing intrinsic transport properties of single metal nanowires: Direct-write contact formation using a focused ion beam, J. Appl. Phys. 96 (2004) 3458-3462. doi:10.1063/1.1779972.

[8] R.M. Langford, T.-X. Wang, M. Thornton, A. Heidelberg, J.G. Sheridan, W. Blau, et al., Comparison of different methods to contact to nanowires, J. Vac. Sci. Technol. B Microelectron. Nanom. Struct. 24 (2006) 2306. doi:10.1116/1.2348731.

[9] N.P. Dasgupta, J. Sun, C. Liu, S. Brittman, S.C. Andrews, J. Lim, et al., Semiconductor Nanowires--Synthesis, Characterization, and Applications., Adv. Mater. 26 (2014) $2137-$ 84. doi:10.1002/adma.201305929.

[10] Y. Cui, Q. Wei, H. Park, C.M. Lieber, Nanowire Nanosensors for Highly Sensitive and Selective Detection of Biological and Chemical Species, Science (80-. ). 293 (2001) 1289-1292.

[11] F. Patolsky, G. Zheng, C.M. Lieber, Nanowire-based biosensors, Anal. Chem. Chim. Acta. 78 (2006) 4260-4269. 
[12] C. Soci, A. Zhang, X.Y. Bao, H. Kim, Y. Lo, D. Wang, Nanowire photodetectors, J. Nanosci. Nanotechnol. 10 (2010) 1430-1449.

[13] Y. Cui, C.M. Lieber, Functional Nanoscale Electronic Devices Assembled Using Silicon Nanowire Building Blocks, Science (80-. ). 291 (2001) 851-853.

[14] J. Goldberger, D.J. Sirbuly, M. Law, P. Yang, ZnO nanowire transistors., J. Phys. Chem. B. 109 (2005) 9-14. doi:10.1021/jp0452599.

[15] F. Hernandez-Ramirez, J.D. Prades, R. Jimenez-Diaz, T. Fischer, A. Romano-Rodriguez, S. Mathur, et al., On the role of individual metal oxide nanowires in the scaling down of chemical sensors., Phys. Chem. Chem. Phys. 11 (2009) 7105-7110. doi: $10.1039 / \mathrm{b} 905234 \mathrm{~h}$.

[16] Y. Zhang, a. Kolmakov, S. Chretien, H. Metiu, M. Moskovits, Control of catalytic reactions at the surface of a metal oxide nanowire by manipulating electron density inside it, Nano Lett. 4 (2004) 403-407. doi:10.1021/n1034968f.

[17] P. Nguyen, H.T. Ng, T. Yamada, M.K. Smith, J. Li, J. Han, et al., Direct integration of metal oxide nanowire in vertical field-effect transistor, Nano Lett. 4 (2004) 651-657. doi:10.1021/n10498536.

[18] S.Y. Lee, A. Umar, D. Il Suh, J.E. Park, Y.B. Hahn, J.Y. Ahn, et al., The synthesis of ZnO nanowires and their subsequent use in high-current field-effect transistors formed by dielectrophoresis alignment, Phys. E Low-Dimensional Syst. Nanostructures. 40 (2008) 866-872. doi:10.1016/j.physe.2007.10.094.

[19] E. Comini, G. Sberveglieri, Metal oxide nanowires as chemical sensors, Mater. Today. 13 (2010) 36-44. doi:10.1016/S1369-7021(10)70126-7.

[20] J.R. Morante, Chemical to electrical transduction mechanisms from single metal oxide nanowire measurements: response time constant analysis., Nanotechnology. 24 (2013) 444004. doi:10.1088/0957-4484/24/44/444004.

[21] F. Hernandez-Ramirez, J.D. Prades, A. Tarancon, S. Barth, O. Casals, R. Jimenez-Diaz, et al., Insight into the Role of Oxygen Diffusion in the Sensing Mechanisms of SnO 2 Nanowires, Adv. Funct. Mater. 18 (2008) 2990-2994. doi:10.1002/adfm.200701191.

[22] E. Strelcov, S. Dmitriev, B. Button, J. Cothren, V. Sysoev, A. Kolmakov, Evidence of the self-heating effect on surface reactivity and gas sensing of metal oxide nanowire chemiresistors., Nanotechnology. 19 (2008) 355502. doi:10.1088/0957$4484 / 19 / 35 / 355502$.

[23] J.D. Prades, R. Jimenez-Diaz, F. Hernandez-Ramirez, S. Barth, A. Cirera, A. RomanoRodriguez, et al., Ultralow power consumption gas sensors based on self-heated individual nanowires, Appl. Phys. Lett. 93 (2008) 123110. doi:10.1063/1.2988265. 
[24] J.D. Prades, R. Jimenez-Diaz, F. Hernandez-Ramirez, J. Pan, a. Romano-Rodriguez, S. Mathur, et al., Direct observation of the gas-surface interaction kinetics in nanowires through pulsed self-heating assisted conductometric measurements, Appl. Phys. Lett. 95 (2009) 053101. doi:10.1063/1.3192358.

[25] H. Kind, H. Yan, B. Messer, M. Law, P. Yang, Nanowire ultraviolet photodetectors and optical switches, Adv. Mater. 14 (2002) 158-160. doi:10.1002/15214095(20020116)14:2<158::AID-ADMA158>3.0.CO;2-W.

[26] J.D. Prades, F. Hernandez-Ramirez, R. Jimenez-Diaz, M. Manzanares, T. Andreu, A. Cirera, et al., The effects of electron-hole separation on the photoconductivity of individual metal oxide nanowires., Nanotechnology. 19 (2008) 465501.

[27] C. Soci, a Zhang, B. Xiang, S. a Dayeh, D.P.R. Aplin, J. Park, et al., ZnO nanowire UV photodetectors with high internal gain., Nano Lett. 7 (2007) 1003-9. doi:10.1021/n1070111x.

[28] J. Suehiro, N. Nakagawa, S.-I. Hidaka, M. Ueda, K. Imasaka, M. Higashihata, et al., Dielectrophoretic fabrication and characterization of a $\mathrm{ZnO}$ nanowire-based UV photosensor., Nanotechnology. 17 (2006) 2567-2573. doi:10.1088/0957-4484/17/10/021.

[29] S. Mathur, S. Barth, H. Shen, J.-C. Pyun, U. Werner, Size-dependent photoconductance in SnO2 nanowires., Small. 1 (2005) 713-7. doi:10.1002/smll.200400168.

[30] S. Mathur, S. Barth, Molecule-based chemical vapor growth of aligned SnO2 nanowires and branched SnO2/V2O5 heterostructures., Small. 3 (2007) 2070-5. doi:10.1002/smll.200700213.

[31] R. Müller, F. Hernandez-Ramirez, H. Shen, H. Du, W. Mader, S. Mathur, Influence of precursor chemistry on morphology and composition of CVD-grown SnO 2 nanowires, Chem. Mater. 24 (2012) 4028-4035. doi:10.1021/cm300913h.

[32] J. Fan, Y. Hao, A. Cabot, E.M.J. Johansson, G. Boschloo, A. Hagfeldt, Cobalt(II/III) redox electrolyte in $\mathrm{ZnO}$ nanowire-based dye-sensitized solar cells., ACS Appl. Mater. Interfaces. 5 (2013) 1902-6. doi:10.1021/am400042s.

[33] A. Fontcuberta i Morral, J. Arbiol, J.D. Prades, A. Cirera, J.R. Morante, A.F.I. Morral, Synthesis of silicon nanowires with wurtzite crystalline structure by using standard chemical vapor deposition, Adv. Mater. 19 (2007) 1347-+. doi:10.1002/adma.200602318.

[34] T. Iwaki, J.A. Covington, J.W. Gardner, F. Udrea, C.S. Blackman, I.P. Parkin, SQICMOS heaters for gas sensors, IEEE SENSORS. 1-3 (2006) 460-463.

[35] J.D. Prades, R. Jimenez-Diaz, F. Hernandez-Ramirez, S. Barth, J. Pan, A. Cirera, et al., An experimental method to estimate the temperature of individual nanowires, Int. J. Nanotechnol. 6 (2009) 860-869. 
[36] O. Bierwagen, M.E. White, M.Y. Tsai, T. Nagata, J.S. Speck, Non-alloyed schottky and ohmic contacts to as-grown and oxygen-plasma treated n-type SnO2 (110) and (101) thin films, Appl. Phys. Express. 2 (2009) 1-4. doi:10.1143/APEX.2.106502.

[37] O. Harnack, C. Pacholski, H. Weller, A. Yasuda, J.M. Wessels, Rectifying behavior of electrically aligned $\mathrm{ZnO}$ nanorods, Nano Lett. 3 (2003) 1097-1101. doi: $10.1021 / \mathrm{n} 1034240 \mathrm{z}$.

[38] Y. Keun Lee, J. Lee, H. Lee, J.Y. Lee, J. Young Park, Probing polarization modes of Ag nanowires with hot electron detection on $\mathrm{Au} / \mathrm{TiO} 2$ nanodiodes, Appl. Phys. Lett. 102 (2013). doi:10.1063/1.4799156.

[39] F. Hernandez-Ramirez, A. Tarancon, O. Casals, E. Pellicer, J. Rodriguez, A. RomanoRodriguez, et al., Electrical properties of individual tin oxide nanowires contacted to platinum electrodes, Phys. Rev. B. 76 (2007) 085429. doi:10.1103/PhysRevB.76.085429.

[40] C. Fàbrega, F. Hernández-Ramírez, J.D. Prades, R. Jiménez-Díaz, T. Andreu, J.R. Morante, On the photoconduction properties of low resistivity $\mathrm{TiO} 2$ nanotubes., Nanotechnology. 21 (2010) 445703. doi:10.1088/0957-4484/21/44/445703.

[41] N. Yamazoe, K. Shimanoe, Theory of power laws for semiconductor gas sensors, Sensors Actuators B Chem. 128 (2008) 566-573. doi:10.1016/j.snb.2007.07.036.

[42] O. Monereo, J.D. Prades, A. Cirera, Self-heating effects in large arrangements of randomly oriented carbon nanofibers: application to gas sensors, Sensors Actuators B Chem. 211 (2015) 489-497. doi:10.1016/j.snb.2015.01.095.

[43] J.D. Prades, F. Hernández-Ramírez, T. Fischer, M. Hoffmann, R. Mu $\square$ ller, N. López, et al., Quantitative analysis of CO-humidity gas mixtures with self-heated nanowires operated in pulsed mode, Appl. Phys. Lett. 97 (2010) 243105. doi:10.1063/1.3515918.

[44] G. Korotcenkov, Practical aspects in design of one-electrode semiconductor gas sensors: Status report, Sensors Actuators, B Chem. 121 (2007) 664-678. doi:10.1016/j.snb.2006.04.092.

[45] J.D. Prades, R. Jimenez-Diaz, F. Hernandez-Ramirez, L. Fernandez-Romero, T. Andreu, A. Cirera, et al., Toward a Systematic Understanding of Photodetectors Based on Individual Metal Oxide Nanowires, J. Phys. Chem. 112 (2008) 14639-14644. doi: $10.1021 / \mathrm{jp} 804614 \mathrm{q}$.

[46] Y. Liu, C.R. Gorla, S. Liang, N. Emanetoglu, Y. Lu, H. Shen, et al., Ultraviolet detectors based on epitaxial ZnO films grown by MOCVD, J. Electron. Mater. 29 (2000) 69-74. doi:10.1007/s11664-000-0097-1.

[47] P. Chang, Z. Fan, C. Chien, High-performance $\mathrm{ZnO}$ nanowire field effect transistors, Appl. Phys. .... 89 (2006) 133113. doi:10.1063/1.2357013. 
[48] F.J. Arregui, ed., Sensors Based on Nanostructured Materials, Springer-Verlag, 2009. 
Jordi Guilera Sala was born in Vilafranca del Penedès, Spain, in 1986. He received his degree in Chemical Engineering in 2009 at the Universitat de Barcelona, the master degree in Environmental Engineering in 2010 and his PhD in 2013 from the same university. He joined at the Catalonia Institute for Energy Research (IREC) as a postdoctoral researcher during 20132014. Currently, he is lecturer of Chemical Engineering at the Universidad San Francisco de Quito. He has experience on the application of advanced materials for clean and sustainable energy processes.

Cristian Fàbrega was born in Barcelona in 1980, Spain. He graduated in Physics at the University of Barcelona and obtained his Ph.D. at the same institution in 2011. He has experience in material engineering for electrochemical energy storage and specially, in photoelectrochemical hydrogen production. He has also experience in the field of sensors devices. He has published more than 20 papers in peer-reviewed journals. He has also actively involved in several industrial project which resulted in an industrial patent.

O. Casals was born in Barcelona in 1973. She received her diploma in Optics and Optometry at the Polytechnic University of Catalonia in 1994 and her degree in Physics at the University of Barcelona in 2001, and her PhD in 2012. Her professional experience is set in development of new technologies on solid-state gas sensors.

Francisco Hernández-Ramírez graduated in Physics at the University of Barcelona in 2003 and obtained his Ph.D. at the same institution in 2007. He is actively involved in the development of innovative sensor prototypes based on nanowires. He has published more than 50 papers in peer-reviewed journals and contributed to five industrial patents.

Shuangzhou Wang is a PhD student at the Institute of Inorganic Chemistry at the University of Cologne in Germany.

Sanjay Mathur is the Director of the Institute of Inorganic Chemistry at the University of Cologne in Germany. He is the Director of the Institute of Renewable Energy Sources at the Xian Jiao Tong University, Xian, China and a World Class University Professor at the Chonbuk University in Korea. He also holds Visiting Professorships at the Central South University, China and National Institute of Science Education and Research (NISER), India. His research interests focus on various facets of chemical nanotechnologies with thrust on chemical processing of functional nanostructures for diversified applications ranging from catalysis, solar hydrogen production, nanotoxicology studies, engineered surfaces, and devices for energy applications, where he holds six patents. He has edited twelve books and has authored/co-authored over 300 original research publications and several book chapters.

Florian Udrea is Professor of Semiconductor Engineering at Cambridge University with over 20 years' experience in power devices, smart technologies, micro-sensors, and MEMS. Prof. Florin Udrea is an inventor of over 70 patents. For his 'outstanding contribution to British Engineering' he has received the Silver Medal from the Royal Academy of Engineering for 2012. Since joining Cambridge University in 1992, he has worked on industrial projects with several major European, American and Japanese-based multinationals and has been a consultant to a number of design companies and semiconductor foundries. He is also a cofounder of CamSemi and Cambridge CMOS Sensors. 
Andrea De Luca graduated from the University of Naples Federico II (Naples, Italy) with a degree in Electronic Engineering in 2011, and is currently a Ph.D. student at the University of Cambridge (Cambridge, UK). His research interests include temperature sensors, thermal flow sensors, infrared emitters and detector for non-dispersive-infra-red spectroscopy, microhotplate based gas sensors and nanomaterials for infrared absorption/emission enhancement and gas sensing. He is author or co-author of 15 publications in journals and conferences, and co-inventor of one patent application.

Syed Zeeshan Ali graduated from GIK Institute (Pakistan) in 2003 with a BS in electronic engineering. He completed his PhD in 2007 at the University of Cambridge (UK) on the design of micro-hotplates for smart gas sensors and electro-thermo-mechanical modelling of membrane devices and was then a research associate at the University of Cambridge researching on micro-hotplates and gas sensing materials. He joined Cambridge CMOS Sensors (UK) in 2010 and is at present an R\&D Leader at the company. His areas of research are microsensors and MEMS devices including gas sensors, IR emitters and IR detectors. He is the author or co-author of over 50 publications in international journals and conferences.

A. Romano-Rodríguez received his degree in Physics at the University of Barcelona in 1986. He immediately joined the group of Electronics at the Department of Applied Physics and Electronics (FAE) of the same university. In 1989 he joined the laboratory IMEC (Leuven, Belgium), where he worked out his PhD in Physics, presented at the University of Barcelona (1991), on the application of electron microscopy for the development of ULSI devices. He continued in IMEC as post-doctoral fellow and returned to UB as researcher in 1991 and was appointed in 1993 as Professor Titular in electronics. His research activities are in the fields of structural, chemical and electrical characterisation of electronic materials and processes employed in the electronic industries and in the development, characterisation and test of chemical sensors. Dr. Romano has co-authored over 70 papers in international journals and over 120 contributions to conferences.

J. Daniel Prades was born in Barcelona in 1982. He graduated in Physics and Electronic Engineering at the University of Barcelona and obtained his Ph.D. at the same institution in 2009. He has experience in modelling of the electronic and vibrational properties of nanostructured metal oxides and in their experimental validation. He is actively involved in the development of innovative device prototypes based on nanomaterials. He has published more than 50 papers in peer-reviewed journals and contributed to more than 150 international conferences. He has also contributed to five industrial patents.

Joan R. Morante is the head of the Advanced Materials for Energy Applications Area of the Catanlonian Institute of Energy Research (IREC). He has co-authored more than 500 works in international specialized journals, ( $h=58$ Scopus data bases), with more of 12.800 citations, several patents (15) and he has also been advisor of more than $35 \mathrm{PhD}$ students; he is member of international committees and editorial boards of scientific journals. He has been general chairman of international events and has been guest editor of many special issues. He has been awarded the Narcís Monturiol of the Generalitat of Catalunya, and the "senior research distinction" of the Generalitat of Catalunya. 